\title{
Foreign bodies in the lung: three sewing needles
}

\section{Case report}

A 32-years old male came to our observation with cough and hemoptysis that developed in the last month. During the past eleven years the patient presented periodically severe coughing attacks even though he stayed for long periods symptoms. The anamnesis showed that he had worked in the past in a textile factory and he also reported a working accident, and although he didn't accurately remember the fact it appears that he had a blackout and woke up in hospital. He didn't give us any other detail on this event.

The chest roentgenogram at admission showed the presence of three foreign bodies (Figure 1A \& Figure 1B) into an area of chronic inflammatory disease in the right middle lobe of the lung. A successive bronchoscopy was negative for intrabroncheal foreign bodies, but showed the presence of coagulated blood in the middle bronchus of the right lung.

The patient underwent a right anthero-lateral thoracotomy, and after debridement of pleuroparenchymal adhesion and exposition of middle right lobe, the presence of three foreign bodies was confirmed. At the end of the surgical procedure three sewing needles (Figure 2) were removed from that area, and after having assessed the integrity
Volume 4 Issue 2 - 2017

\author{
Antonella Lombardi,' Stefano Bongiolatti,, \\ Maurizio Di Bisceglie' \\ 'General Surgery, MD- Misericordia Hospital, Italy \\ ${ }^{2}$ Chirurgia Toracica - AUC Careggi, Italy
}

Correspondence: Antonella Lombardi, Misericordia Hospital, Department of Surgery, VIA Senese 169, Grosseto, 58100, Tel 003-905-644-853-53, Email antonellalomb@virgilio.it

Received: October 27, 2017 | Published: March 07, 2017

of the three foreign bodies, the procedure was completed with an atypical resection of the middle lobe that included the chronic parenchymal inflammation and a chest tube was positioned in the pleural cavity. On the second postoperative day a chest roentgenogram demonstrated good pulmonary re-expansion and the chest tube was removed. The patient was discharged on postoperative day 5 in good general conditions and was followed in the out-patients clinic until he presented excellent general conditions.
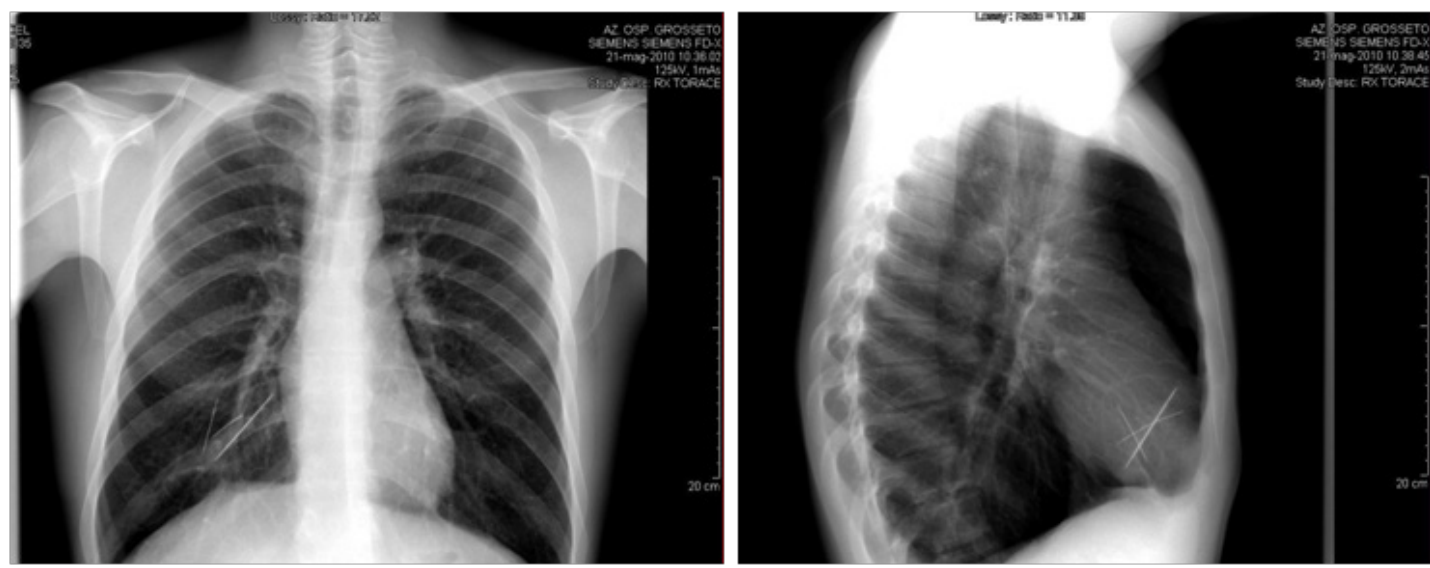

Figure IA-B The chest roentgenogram at admission showed the presence of three foreign bodies (Figure IA-B) into an area of chronic inflammatory disease in the right middle lobe of the lung.

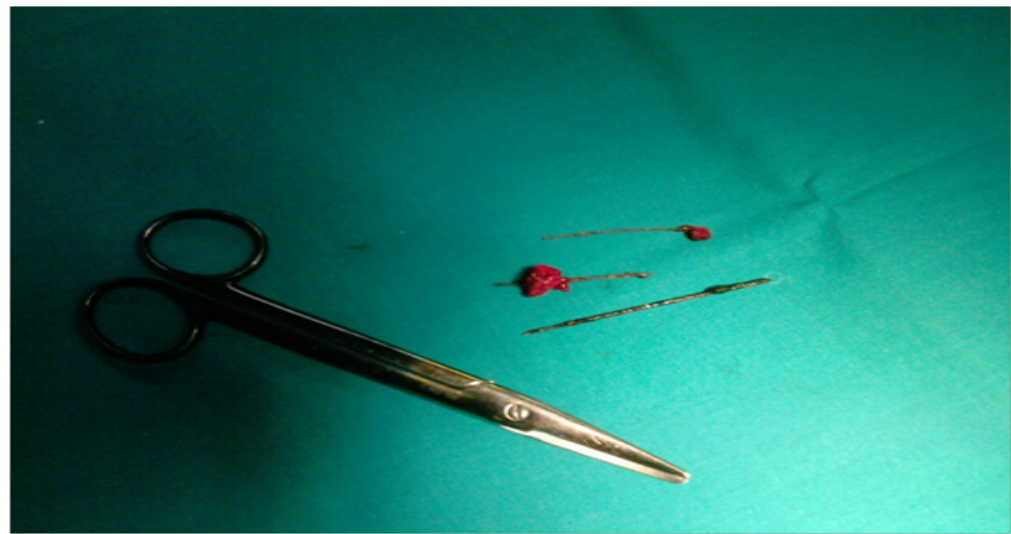

Figure 2 Three sewing needles removed, were located in the middle right lobe. 


\section{Discussion}

After the evalutation of clinical case the question is if in patient with sewing needle injuries in the lung, is the surgical approach the best treatment? The scientific literature was reviewed by searching medline, but anything paper was found about the sewing needle injuries in the lung. Was reported some cases of intra-thoracicmigration of acupunctureneedles or foreignneedlesingest, All clinical report of needle injuries in the parenchyma, regarding the cardiac area and in nearly all cases the authors surgically removed the needles from the heart, to prevent migration and further anatomical damage. In our case, due to the tendency of the needle to migrate and the prolonged symptoms, the surgical treatment may be the best choice. If the symptoms are less severe it is reasonable to adopt a conservative treatment as with time most foreign bodies become safely encysted and do no harm. In case of conservative treatment, a strict follow-up is useful in those patients. ${ }^{1-4}$

\section{Conclusion}

After a literature's review, anything manuscript similar was found; all cases reported in literature of needle injuries regarded the cardiac area and in nearly all cases the authors surgically removed the needles from the heart, to prevent migration and further anatomical damage. If the lung's location suggests a conservative treatment, however the prolonged symptom and the progressive migration, indicated a surgical treatment. Requiring an intervention of atypical resection of lung for chronic parenchymal inflammation.

\section{Acknowledgements}

None.

\section{Conflict of interest}

The author declares no conflict of interest.

\section{References}

1. J Kim DH, Kim SC, Youn HC. Surgical treatment for intra-thoracicmigration of acupunctureneedles. Korean Med Sci. 2012;27(3):281-284.

2. Von Riedenauer WB, Baker MK, Brewer RJ. Video-assisted thorascopic removal of migratory acupuncture needle causing pneumothorax. Chest. 2007;131(3):899-901.

3. Choudhary S, PujarVenkateshacharya S, Reddy C. Sewingneedle: a rare cause of intra-cardiacforeign body in a 3-year-old child. Cardiol Young. 2016;26(7):1425-1427.

4. Actis Dato GM, Arslanian A, Di Marzio P, et al. Posttraumatic and iatrogenicforeignbodies in the heart: report of fourteencases and review of the literature. J Thorac Cardiovasc Surg. 2003;126(2):408-414. 